\title{
Efficacy of Carbon Microcoils in Relieving Cervicogenic Dizziness
}

\author{
Roseli Bittar ${ }^{1}$ Nédison Gomes Paim Alves ${ }^{1} \quad$ César Bertoldo $^{1} \quad$ Cibele Brugnera $^{1} \quad$ Jeanne Oiticica ${ }^{1}$ \\ 1 Department of Otorhinolaryngology, Universidade de São Paulo, \\ São Paulo, SP, Brazil \\ Int Arch Otorhinolaryngol 2017;21:4-7. \\ Address for correspondence Jeanne Oiticica, MD, PhD, Department of \\ Otorhinolaryngology, Universidade de São Paulo, Alameda dos Jurupis, \\ 657 CJ 72, Indianópolis, Sao Paulo, SP 04088002, Brazil \\ (e-mail: jeanneramalho@uol.com.br).
}

\section{Introduction}

Dizziness is a common condition that occurs in people of all ages. ${ }^{1}$ A recent domiciliary survey in the city of São Paulo reported an incredible dizziness prevalence of $42 \%$ in the population. ${ }^{2}$ The medical diagnosis of dizziness is often challenging because of its varied etiologies and manifestations, one of which is cervicogenic dizziness.

Cervicogenic dizziness arises from the abnormal afferent inputs from the proprioceptors of the extensor muscles and joints of the neck, as well as from localized, immediate, and sustained pain. ${ }^{3}$ These conflicting signals diverge from visual and vestibular network in the central nervous system (CNS). ${ }^{4,5}$ This type of unmatched balance integration in the CNS is experienced as dizziness. In our clinical experience, the most common complaints during episodes of dizziness related to cervical pain are disorientation and mild instability, which can last from minutes to hours and increase with cervical movement and/or worsening of neck pain. We also observe a temporal relationship between dizziness and neck pain, as well as an association between the relief of cervical pain and relief from dizziness. Some patients with cervical pain may also report visual conflict and falls. ${ }^{6,7}$ Neck pathology as trauma, arthritis, whiplash, articular block, trigger received

May 19, 2016

accepted

August 14, 2016

published online

October 26, 2016
DOI http://dx.doi.org/

10.1055/s-0036-1592418. ISSN 1809-9777.
Copyright (c) 2017 by Thieme-Revinter

Publicações Ltda, Rio de Janeiro, Brazil
License terms

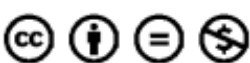




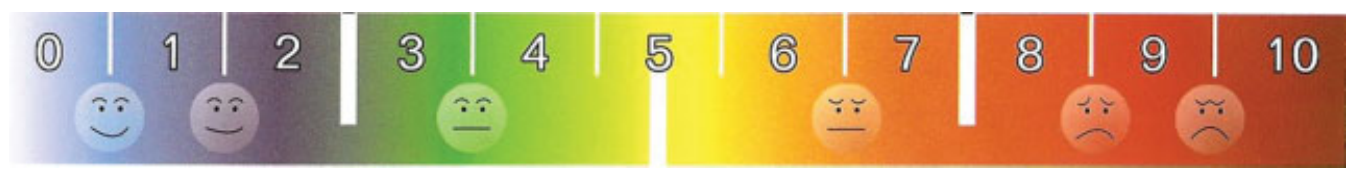

Fig. 1 "Visual Analogue Scale - VAS" presented to the patient.

points, tender points, and disc herniation are the common etiological diagnoses.

The prevalence of chronic pain in the city of São Paulo is $28.7 \%$ and $22.1 \%$ of these cases are associated with "spinal problems". ${ }^{8}$ According to Giamberardino et al, musculoskeletal pain affects $85 \%$ of the population at some point in their lives. Myofascial pain is the most common form of musculoskeletal pain. ${ }^{9}$ Cervical pain is a type of myofascial pain and is treated using several different well-documented techniques. Among the non-pharmacologic techniques, there are those that use heat as a therapeutic strategy, such as the use of thermal bags, ultra-sound, paraffin baths, infrared light, bier ovens, or whirlpool hydrotherapy and hydromassage bathtubs. The use of nanotechnology stands out among newer thermotherapy-based techniques developed for the treatment of neck pain. ${ }^{10}$ Nanotechnology could be used for the treatment of neck pain by fixing to the skin small rounds silicone patches containing double spiral carbon nanotubes arranged in the form of a coil (Helical, Miyasaka Rubber Co., Chino, Nagano, Japan). These patches are designed for home use, made of an inert material, do not contain any type of drugs, and can be purchased without a medical prescription. When placed over the skin, the patches absorb the electromagnetic waves from the tense muscles, find resistance as they flow through the carbon nanotubes, induce heat, and lead to muscle relaxation. ${ }^{11}$ We thus expect that this treatment could ease neck pain, therefore relieving dizziness from muscular contraction. Our aims were to evaluate pain and dizziness scores before and after Helical patches, as well as their effect on cervicogenic dizziness treatment.

\section{Method}

Here we present a series of case studies approved by the ethics committee of our institution under the number 1.433 .854 from March $2^{\text {nd }}, 2016$. We selected eight patients diagnosed with cervicogenic dizziness from the Neurotology Outpatient Day Clinic of Hospital das Clínicas, Universidade de São Paulo.

The patients underwent a physical evaluation of the cervical region. The same therapist performed all examinations. For the initial evaluation, patients were placed in supine position on a stretcher, with the therapist sitting behind their head. The therapist performed neck and shoulder manual palpation to identify tender areas, as well as muscle contractions. The mobility of the cervical spine was tested in the pitch axis using the Maitland technique. ${ }^{12}$

Our inclusion criteria were: 1) dizziness with loss of balance or instability lasting more than 90 days, 2) neck pain arising from muscle contraction and 3 ) normal electro- oculography. Our exclusion criteria were 1) psychiatric disease, 2) neurological disease, 3) orthopedic disease, 4) cognitive deficits 5) neck pain from articular etiology and 6 ) the use of drugs for the treatment of pain or dizziness. The selected patients received guidance on all procedures throughout the study length and provided informed consent.

Patients reported their pain and dizziness levels before, during, and after the treatment using a Visual Analogue Scale (VAS), where the absence of symptoms corresponded to a zero score and the maximum bearable intensity was scored as 10 (-Fig. 1).

The treatment consisted of placing 10 Helical patches spread as follows (-Fig. 2):

- Two in the upper cervical area (suboccipital);

- Two in the lower cervical area (near the $5^{\text {th }}$ and $6^{\text {th }}$ vertebrae);

- Two in the upper trapezius muscle area (between neck and shoulder);

- Four in the tender point area (as reported by the patient).

In a preliminary study conducted before this one, we noticed a decrease in pain and dizziness scores throughout two weeks of patches treatment. Therefore, we tracked out the patient on a biweekly basis. We changed the patches during the first visit, which was 15 days after the beginning of treatment. At this point, one of the subjects was excluded from the study due to patch loss. We recorded the remaining seven subjects pain and dizziness levels using the VAS on a twice week basis. A Computerized Dynamic Posturography (CPD) was performed at the end of four weeks. We plotted sex, age, pain, and dizziness scores in a table. We analyzed

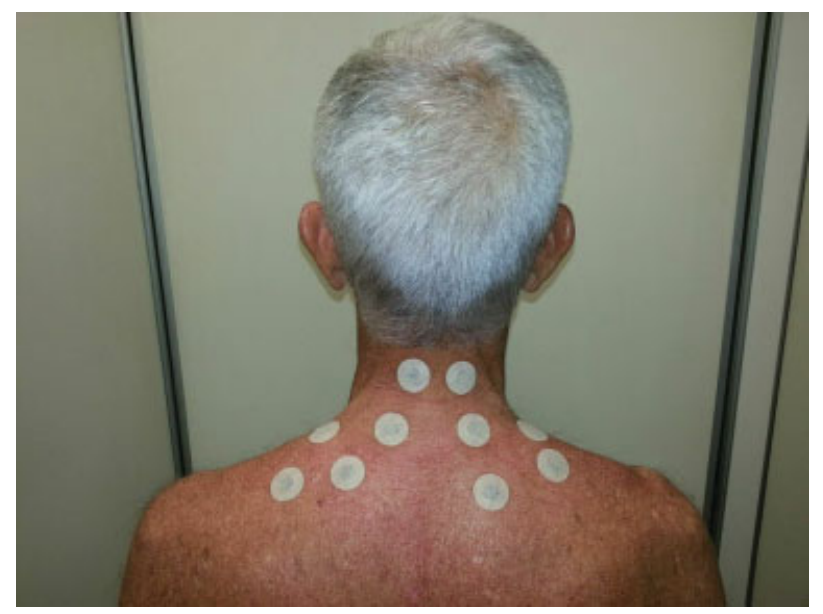

Fig. 2 Distribution of the 10 tablets containing nanotubes. 
Table 1 Individual pain and dizziness scores on the first, fifteenth, and thirtieth days of the experiment

\begin{tabular}{|l|l|l|l|l|l|l|l|l|}
\hline \multirow{2}{*}{ Patients } & \multirow{2}{*}{ Sex } & \multirow{2}{*}{ Age } & \multicolumn{2}{|l|}{ Day 1} & \multicolumn{2}{l|}{ Day 15 } & \multicolumn{2}{l|}{ Day 30} \\
\cline { 3 - 8 } & & & Pain & Dizziness & Pain & Dizziness & Pain & Dizziness \\
\hline NFS & $\mathrm{F}$ & 67 & 10 & 6 & 5 & 2 & 8 & 3 \\
\hline CBR & $\mathrm{M}$ & 59 & 8 & 7 & 4 & 3 & 3 & 2 \\
\hline RRRC & $\mathrm{F}$ & 54 & 8 & 8 & 4 & 4 & 5 & 0 \\
\hline JALL & $\mathrm{F}$ & 50 & 5 & 8.5 & 6 & 0 & 9 & 8 \\
\hline CMPA & $\mathrm{F}$ & 64 & 8 & 9 & 3 & 0 & 4 & 0 \\
\hline LN & $\mathrm{F}$ & 74 & 8 & 8 & 7 & 8 & 4 & 6 \\
\hline JMC & $\mathrm{M}$ & 57 & 5 & 5 & 3 & 5 & 3 & 3 \\
\hline \multirow{2}{*}{ Mann-Whitney Test } & & $\mathrm{Z}$ & & $2.43^{*}$ & $2.62^{*}$ & 1.66 & $2.36^{*}$ \\
\cline { 2 - 9 } & & $\mathrm{U}$ & $5^{*}$ & $3.5^{*}$ & 11 & $5.5^{*}$ \\
\hline
\end{tabular}

The $\mathrm{Z}$ and $\mathrm{U}$ indexes for $p \leq 0.05$ of a two-tailed curve are also calculated. Asterisk $\left({ }^{*}\right)$ indicates statistical significance. Note: Results of the Mann-Whitney test comparing data from day 1 to day 15 and from day 1 to day 30 are presented.

data by mean and standard deviation or using Mann-Whitney test depending on variable category. We also generated a box plot with median, maximum, and minimum values, $(p<0.05)$.

\section{Result}

Our sample consisted of five women and two men, with ages varied between 50 and 74 years (mean and standard deviation $=60.71 \pm 8.19$ ). VAS ratio scores recorded on day 1 and 15 and day 1 and 30 are summarized in -Table 1 .

We matched pain and dizziness scores from the day 1 to those from day 15 and day 30 using Mann-Whitney test for small samples. There was a significant difference between pain score reported on day 1 and $15(Z=2.43, U=5$, $p=0.01)$. However, no such difference was found when comparing the day 1 and $30(\mathrm{Z}=1.66, \mathrm{U}=11, p=0.09)$ (-Table 1). The results are present as a box plot (-Fig. $\mathbf{3}$ ).

We also found significant differences between dizziness scores reported at days 1 and $15(\mathrm{Z}=2.62, \mathrm{U}=3.5, p=0.01)$ and days 1 and $30(\mathrm{Z}=2.36, \mathrm{U}=5.5, p=0.01)$ [ Table 1]. The results appear as a box plot (-Fig. 4).

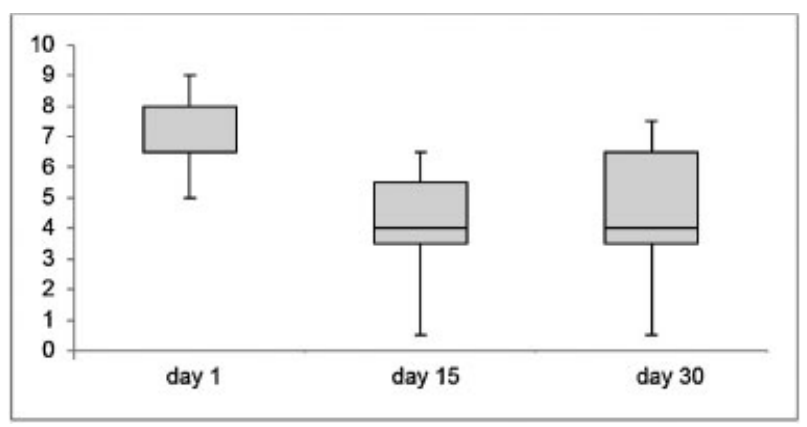

Fig. 3 Box plot of the VAS pain scores reported during the study period.
We observed no side effects, such as itching or hyperemia, in our patients. However, one of the patients lost a patch and was excluded from the sample. None of the patients used pain relief medication during the time treatment.

\section{Discussion}

Every year, 11 to $14 \%$ of the economically active population report functional limitations and missing work due to neck pain. ${ }^{13}$ Risk factors include repetitive work, bad postural habits, stress, smoking, and previous trauma. The resulting cervical injury contributes to postural deviations and to anterior-posterior imbalance in the affected subjects. ${ }^{13}$

The relationship between the cervical spine and vestibular pathways is well-documented in the literature.6,14,15 Many therapies are reportedly beneficial for neck pain relief and, thus, for dizziness amelioration. However, none of the previous reported therapies combines low cost, low side effects, and sustains improvement. ${ }^{6,14,15}$ We decided to analyze the efficacy of carbon nanotube patches (Helical) for the treatment of cervicogenic dizziness given that it does not contain drugs, is feasible to use, and known to be effective on neck pain relief. ${ }^{10}$

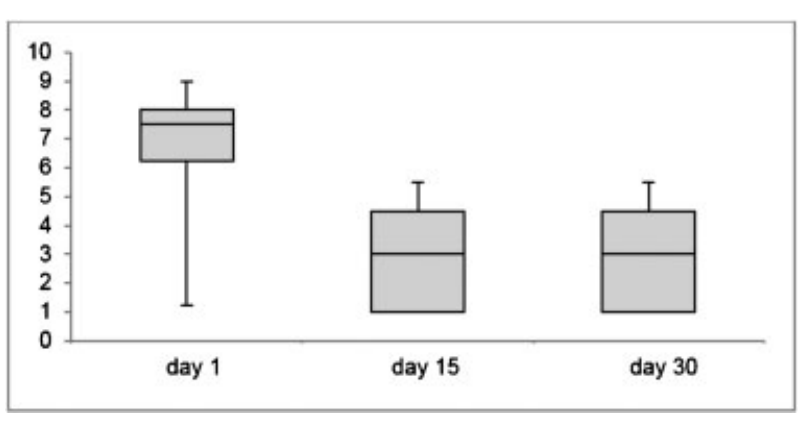

Fig. 4 Box plot of the VAS dizziness score reported during the study period. 
The double-helical carbon nanotubes within the patches are structures capable of absorbing electromagnetic waves. When in contact with the skin of the affected muscle, the heat elicited from the nanotubes is able to restore local blood flow and tissue homeostasis, as well as oxygenate, and reestablish normal muscle activity. We attached the patches over tender skin areas as well as acupuncture points, using an adhesive tape. ${ }^{11,16}$ According to the manufacturer, the patient may experience local mild itching and eczema, side effects that we did not observe in our study.

The average age of our sample is comparable to that previously reported for patients with cervicogenic dizziness, who are usually in the fifth to sixth decade of life. The pain and dizziness scores are usually described as moderate to severe. Our sample reached a 7.5 mean VAS score, corroborating our previously clinical judgement. Prior clinical trial study reported a significant decrease in pain VAS scores three weeks after the patches treatment. ${ }^{10}$ In the current study, we measured pain scores at two time points: 2 and 4 weeks after the beginning of the treatment. We notice that relief of the symptoms occurred in six subjects (86\%), on treatment day 15 $(p \leq 0.01)$. After 30 days, three subjects reported an increase in pain scores compared with day 15 . However, they still had lower pain scores compared with those on day $1(p \leq 0.09)$. Our results suggest that the patches have their best analgesic peak around 15 days after treatment onset, at which time they should be change (-Fig. $\mathbf{3}$ ).

Our main goal was to evaluate effectiveness of the patches on cervicogenic dizziness. We noticed a significant relief on dizziness symptoms both after day $15(p=0.01)$ and day 30 $(p=0.01)$. Three subjects $(42 \%)$ reported dizziness scores improvement after day 15 and sustained it until day 30. Two subjects (28\%) reported dizziness relief only by day 30 . Two patients $(28 \%)$ noticed worsening on dizziness scores between the day 15 and 30, after symptoms relief in the first two weeks. It seems that patch effects on dizziness scores remained even after the analgesic peak (-Fig. 4).

\section{Conclusion}

The Helical patches seem to be an effective treatment for cervicogenic dizziness. The most hopeful aspects of carbon nanotubes are that they do not contain any drugs and that they act by mild heat rise. We believe that the main asset of this therapy is that it provides sustained heat flow, which results in tension muscles relaxation. The absence of side effects in our sample suggests good use tolerance. Our sample consisted of a small group of patients and new studies are necessary to verify the efficacy of this new technique.

\section{References}

1 Nunes A, Al-Jamal K, Nakajima T, Hariz M, Kostarelos K. Application of carbon nanotubes in neurology: clinical perspectives and toxicological risks. Arch Toxicol 2012;86(7):1009-1020

2 Bittar RSM, Oiticica J, Bottino MA, Ganança FF, Dimitrov R. Population epidemiological study on the prevalence of dizziness in the city of São Paulo. Braz J Otorhinolaryngol 2013;79(6): 688-698

3 Fischer AJ, Verhagen WI, Huygen PL. Whiplash injury. A clinical review with emphasis on neuro-otological aspects. Clin Otolaryngol Allied Sci 1997;22(3):192-201

4 Brandt T. Cervical vertigo-reality or fiction? Audiol Neurootol 1996;1(4):187-196

5 Bracher ES, Almeida CI, Almeida RR, Duprat AC, Bracher CB. A combined approach for the treatment of cervical vertigo. J Manipulative Physiol Ther 2000;23(2):96-100

6 Hülse M, Hölzl M. [Vestibulospinal reactions in cervicogenic disequilibrium. Cervicogenic imbalance]. HNO 2000;48(4): 295-301

7 Treleaven J, Jull G, Sterling M. Dizziness and unsteadiness following whiplash injury: characteristic features and relationship with cervical joint position error. J Rehabil Med 2003;35(1):36-43

8 Ferreira KASL, EPI-dor: retratos da dor no Brasil. . Available at: http://pt.slideshare.net/marcuspai/epi-dor-retratos-dor-brasivalidok. July 18th, 2009

9 Giamberardino MA, Affaitati G, Fabrizio A, Costantini R. Myofascial pain syndromes and their evaluation. Best Pract Res Clin Rheumatol 2011;25(2):185-198

10 Tsuji DH, Awade R, Posso IP. Cutaneous application of silicone wafers containing carbon microcoils: efficacy in relieving chronic muscle tension and pain. J Altern Complement Med 2015;21(7):439-443

11 Motojima S, Noda Y, Hoshiya S, Hishikawa Y. Electromagnetic wave absorption properties of carbon microcoil in $12-110 \mathrm{GHz}$ region. J Appl Phys 2003;94(4):2325-2330

12 Maitland G, Hengeveld E, Banks K, English K, Eds. Maitland's Vertebral Manipulation. Ed 7th. Philadelphia: Elsevier; 2005

13 Côté P, van der Velde G, Cassidy JD, et al. Bone and Joint Decade 2000-2010 Task Force on Neck Pain and Its Associated Disorders. The burden and determinants of neck pain in workers: results of the Bone and Joint Decade 2000-2010 Task Force on Neck Pain and Its Associated Disorders. Spine 2008;33(4, Suppl)S60-S74

14 Field S, Treleaven J, Jull G. Standing balance: a comparison between idiopathic and whiplash-induced neck pain. Man Ther 2008;13(3): 183-191

15 Karlberg M, Magnusson M, Malmström EM, Melander A, Moritz U. Postural and symptomatic improvement after physiotherapy in patients with dizziness of suspected cervical origin. Arch Phys Med Rehabil 1996;77(9):874-882

16 Chen X, Motojima S. Vapor phase preparation and some properties of carbon micro-coil (CMCs). KONA 2006;24:222-226 